\title{
An Eccentric Derivation of the Gravitational-Lens Effect
}

\author{
Yoonsoo Bach Park, Il-Tong Cheon \\ ${ }^{1}$ Faculty of Science, Korea Advanced Institute of Science and Technology, Daejeon, South Korea \\ ${ }^{2}$ Korean Academy of Science and Technology, Seongnam, South Korea \\ Email: itcheon@hotmail.com
}

Received September 15, 2012; revised October 28, 2012; accepted November 5, 2012

\begin{abstract}
The gravitational-lens effect is interpreted in the framework of the Newtonian mechanics. Regarding the photon of energy $h v$ as a corpuscle with a tiny mass of $m_{\gamma}=\frac{h v}{c^{2}}$. We calculate it's path bended by the gravitational force near the surface of the sun. Effects of dark matter have also been evaluated.
\end{abstract}

Keywords: Gravitational-Lens Effect; Curved Space; Dark Matter

\section{Introduction}

The Gravitational-Lens Effect (GLE) provides one of the powerful methods to investigate physical properties of stellar objects. This effect is actually the result derived by the General Theory of Relativity (GTR) and, therefore, dynamical understanding is almost impossible without basic knowledge of the GTR, which is usually taught in the graduate course of university. Nevertheless, it might be instructive to derive the GLE on the basis of the Newtonian mechanics which is founded rather upon our experiential events. Indeed, the bending of the light path is originated in the space-time structure induced by the gravitational field, and, therefore, it may not be understandable in the framework of the classical mechanics. Since the light (photon) does not carry any mass, the bending of its path cannot be taken place by the gravitational force in the classical Newtonian mechanics. However, if the photon is interpreted as a corpuscle with a mass, " $m_{\gamma} "=\frac{E_{\gamma}}{c^{2}}$, where $E_{\gamma}$ is the photon energy and the corpuscle is assumed to move always at the speed of light, $c$, without any variation of $m_{\gamma}$, the path of the photon can be bended by the gravitation of the reference stellar object even in the classical Newtonian dynamics. Along this line, the bending angle of the light passing through near the solar surface shall be calculated and our method of calculation will be extended to the system enveloped in dark matter halo.

\section{Derivation of the Gravitational-Lens Effect}

Let us consider the gravitational-lens effect caused by the sun. When the photon is regarded as a corpuscle with a mass $m_{\gamma}$, the gravitational force of the sun acting on it yields

$$
f=-\frac{G M_{\odot} m_{\gamma}}{r^{2}},
$$

where $G$ is the gravitational constant, $M_{\odot}$ is the solar mass and $r$ is the distance between the sun and the corpuscle. The motion of the corpuscle can be obtained by solving the Newtonian equation of motion [1]. Its orbit is actually hyperbolic.

Instead of taking such a treatment, let us try to derive the hyperbolic orbit of the corpuscle on the basis of the geometric property of the conic section. The eccentricity of the hyperbola is generally defined as

$$
e=\frac{P_{I} F}{P_{I} H_{I}},
$$

where $P_{I} F$ and $P_{I} H_{I}$ are distances of an arbitrary point $P_{I}$ on the hyperbola from the focus and from the directrix, $Q_{I}$, respectively, as is shown in Figure 1. One of two focuses in the hyperbola is assighned by $F$ at which the sun is located. In other words, the trace of the point $P_{I}$ which satisfies the relation (2) draws a hyperbola with the eccentricity $e$. Let $P_{I} F$ be $r(\theta)$ where the angle $\theta$ runs clockwise around the focus $F$, then $P_{I} H_{I}$ is given as

$$
P_{I} H_{I}=s+R_{\odot}-r(\theta) \cos \theta,
$$

where $R_{\odot}=A_{0} F$ is the radius of the sun and $s=B_{I} A_{0}$. Accordingly, Equation (2) yields

$$
e=\frac{r(\theta)}{s+R_{\odot}-r(\theta) \cos \theta} .
$$




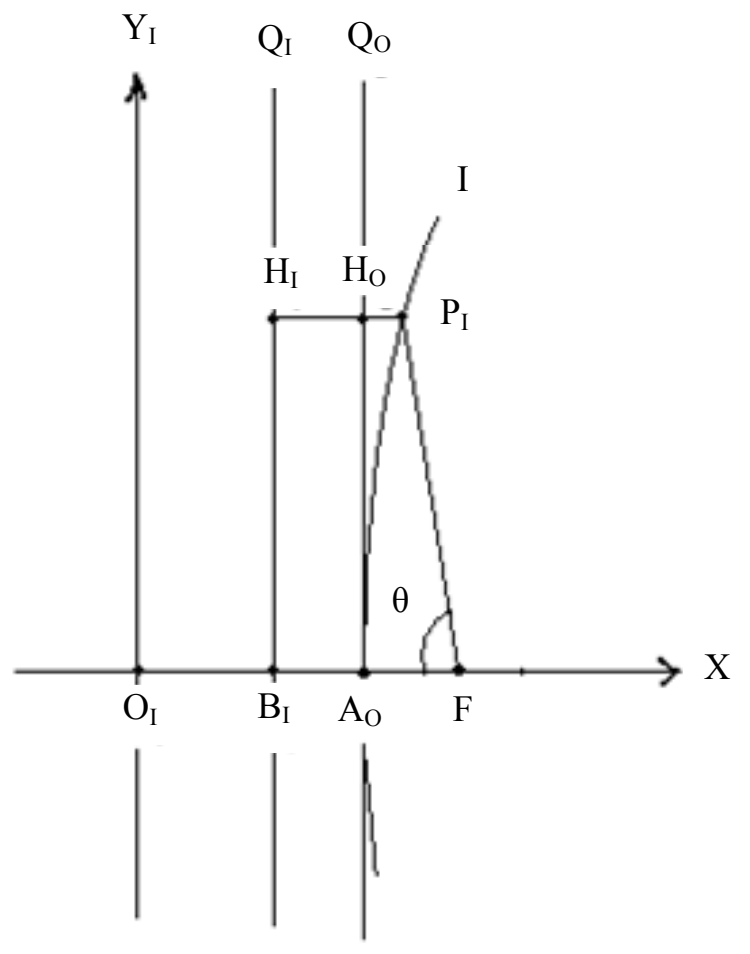

Figure 1. Hyperbolic orbit of a corpuscle. $F$ : focus, $O_{I}$ : the origin, $Q_{I}$ : directrix, $Q_{o}$ : tangent line. The sun is at $F$.

Since $r(\theta=0)=R_{\odot}$ at $\theta=0$, we have

$$
\mathrm{s}=\frac{R_{\odot}}{e} \text {. }
$$

Thus, by solving Equation (4) with respect to $r(\theta)$, the equation of the hyperbola can be obtained in the form

$$
r(\theta)=\frac{R_{\odot}(e+1)}{1+e \cos \theta},
$$

which is exactly identical to the result obtained by solving directly the Newtonian equation of motion [1]. For the particle with its mass, $m_{\gamma}$ moving with the velocity $v$, the angular momentum,

$$
L=m_{\gamma} r v,
$$

is always conserved and the total energy of the system, $E$, is

$$
E=\frac{m_{\gamma} v^{2}}{2}-\frac{k}{r}=\frac{L^{2}}{2 m_{\gamma} r^{2}}-\frac{k}{r},
$$

where $k=G M_{\odot} m_{\gamma}$. The solution of Equation (8) with respect to positive $\frac{1}{r}$ is

$$
\frac{1}{r}=\left(\frac{m_{\gamma} k}{L^{2}}\right)\left[1+\left(1+\frac{2 E L^{2}}{m_{\gamma} k^{2}}\right)^{1 / 2}\right] .
$$

For $v=c$, the angular momentum and the total energy of the system at $\theta=0$ can be obtained by replacing $r$ by $R_{\odot}$ in Equations (7) and (8). Then, reciprocal of the first parenthesis in Equation (9) is equal to the numerator of Equation (6), and, therefore, we find the value of the eccentricity $e$ as

$$
e=\frac{R_{\odot} c^{2}}{G M_{\odot}}-1 .
$$

This result can also be obtained from the second parenthesis in Equation (9). It is easily proved to be $\frac{R_{\odot} C^{2}}{G M_{\odot}}$. And it corresponds to the denominator of Equation (6) at $\theta=0$, which is $(1+e)$. Then, we obtain the same result as that in Equation (10).

On the other hand, the equation of a hyperbola in rectangular coordinates whose origin is located at the center of two focuses can be expressed as

$$
\frac{x^{2}}{a^{2}}-\frac{y^{2}}{b^{2}}=1
$$

A difference between distances of an arbitrary point on the hyperbola from each focus is always equal to $2 a$. This is a basic substance of the hyperbola. From Equation (11), we know immediately that the distance $O A_{o}$ in Figure 1 is $a$. And, thus, the distance between two focuses is $2\left(a+R_{\odot}\right)$. Let $\left(x_{1}, y_{1}\right)$ be the coordinates of a crossing point between the hyperbola and the straight line perpendicular to the $\mathrm{X}$-axis at the focus $\left(x_{1}, 0\right)$. Then, making use of the substance of the hyperbola as well as the Pythagoras theorem, we can easily find

$$
y_{1}=2 R_{\odot}+\frac{R_{\odot}^{2}}{a} .
$$

In addition, from Equation (6), it is obvious that

$$
y_{1}=r\left(\theta=\frac{\pi}{2}\right)=R_{\odot}(e+1) .
$$

Making this result equal to Equation (12) gives $a e=$ $a+R_{\odot}$, which is actually value of $x_{1}$ and, consequently,

$$
a=\frac{R_{\odot}}{e-1} .
$$

Substituting values of $x_{1}=a e$ and $y_{1}=R_{\odot}(e+1)$ in Equation (11), one can find

$$
b=R_{\odot} \sqrt{\frac{e+1}{e-1}} .
$$

Since the equation of the asymptote of the hyperbola is

$$
\frac{x^{2}}{a^{2}}-\frac{y^{2}}{b^{2}}=0,
$$


the angle of the asymptote, $\delta$, is found as

$$
\delta=\arctan \left(\frac{b}{a}\right)=\arctan \sqrt{e^{2}-1} .
$$

Thus, the bending of the light path, i.e. the gravitational-lens effect, is

$$
2 \beta=\pi-2 \delta=\pi-2 \arctan \sqrt{e^{2}-1} .
$$

With values, $R_{\odot}=7 \times 10^{5} \mathrm{~km}, \quad M_{\odot}=2 \times 10^{30} \mathrm{~kg}$, $c=3 \times 10^{5} \mathrm{~km} / \mathrm{s}$ and $G=6.7 \times 10^{-20} \mathrm{~km}^{3} / \mathrm{s}^{2} \cdot \mathrm{kg}$, we obtain

$$
e=4.7 \times 10^{5},
$$

and

$$
2 \beta=0.878^{\prime \prime},
$$

which is in agreement with the result obtained by J. G. von Soldner using a completely different formalization in $1801[2,3]$. However, this result is unfortunately just a half of that obtained in the GTR [4]. The missing factor 2 may be referred to as "relativistic factor".

\section{Newtonian Derivation of the Relativistic Factor 2}

In context of the concept that the structure of the space is associated with the gravitational field, it is convinced that the space around the sun must be curved by its gravitation. Moreover, it should be remarked that the light has nature to travel always along the edge of the space whatever it is straight or curved. The curve $I$ in Figure 2 has been the path of the corpuscle moving under operation of the gravitation. However, if it is interpreted as the edge of the curved space induced by the gravitational field, the photon will travel along this curve $I$ without any influence of the force.

Let us now calculate the path of the corpuscle moving in this curved space when the gravitational force acts directly on it.

As is shown in Figure 2, the directrix $Q_{I}$ in the regular space is now bended into $Q_{N}$ by the same amount of curvature as the edge of the surface of the curved space $I$. Namely, $Q_{N}$ is the translated hyperbola of the curve $I$. Then, the arbitrary point $P_{I}$ on the hyperbola $I$ is shifted to $P_{N}$. Thus, the curve $N$ should be the orbit of the corpuscle when the gravitational force directly acts on it in the curved space. All points, $P_{N}$, $P_{I}, H_{o}, H_{A}$ and $H_{I}$ are on the horizontal line. As the straight line of directrix, $Q_{I}$ is bended into the curve $Q_{N}$ the distance $P_{I} H_{I}$ is shifted to $P_{N} H_{A}$, i.e. $P_{I} H_{I}=P_{N} H_{A}$. When the distance $P_{I} H_{o}$ is expressed by u, i.e. $P_{I} H_{o}=u$, we have, of course, $H_{A} H_{I}=u$, and, therefore, $P_{N} P_{I}$ ought to be $u$. Furthermore, the distance $A_{0} B_{I}$ is expressed by $s$, the distance $P_{I} H_{A}$ should also be $s$, i.e. $P_{I} H_{A}=s$. In accordance with

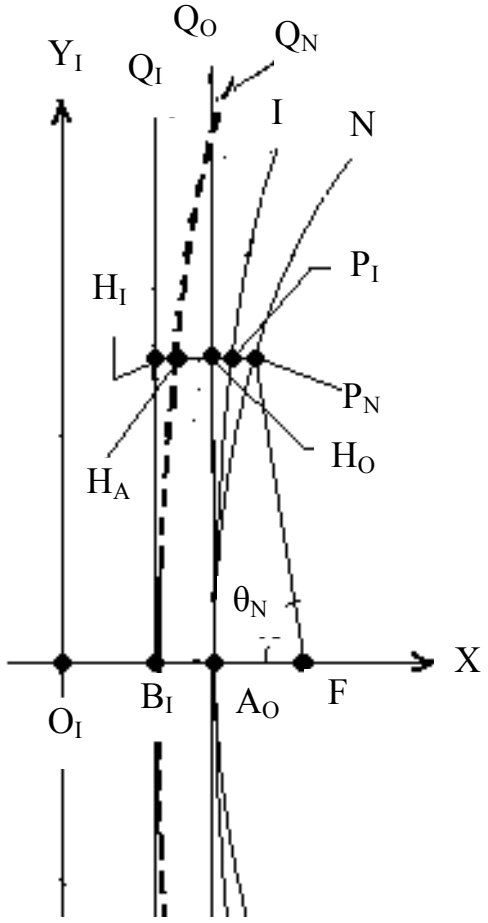

Figure 2. Paths of the corpuscle. $Q_{o}$ : tangent line, $Q_{N}$ : directrix in the curved space, $Q_{I}$ : original directrix.

Equation (2), the eccentricity should be

$$
e=\frac{P_{N} F}{P_{N} H_{A}} \text {. }
$$

When $P_{N} F$ is expressed as $r_{N}\left(\theta_{N}\right)$ and $P_{N} H_{A}=$ $P_{I} H_{A}+P_{N} P_{I}$ is considered, we have

$$
\begin{aligned}
e & =\frac{r_{N}\left(\theta_{N}\right)}{P_{I} H_{A}+P_{N} P_{I}}=\frac{r_{N}\left(\theta_{N}\right)}{s+u} \\
& =\frac{r_{N}\left(\theta_{N}\right)}{\frac{R_{\odot}}{e}+\frac{1}{2}\left[R_{\odot}-r_{N}\left(\theta_{N}\right) \cos \theta_{N}\right]},
\end{aligned}
$$

where $s=\frac{R_{\odot}}{e}$ can be obtained from the second line of Equation (21) because $P_{N} \rightarrow A_{o}$ for $\theta_{N} \rightarrow 0$, and, then, $r_{N}\left(\theta_{N}=0\right)=A_{o} F=R_{\odot}$ and $u=0$. To lead the final expression in Equation (21), we have also used that for $\theta_{N} \neq 0$

$$
P_{N} H_{o}=P_{N} P_{I}+P_{I} H_{o}=2 u=R_{\odot}-r_{N}\left(\theta_{N}\right) \cos \theta_{N} .
$$

Solving Equation (21) with respect to $r_{N}\left(\theta_{N}\right)$, we obtain

$$
r_{N}\left(\theta_{N}\right)=\frac{R_{\odot}\left(\frac{e}{2}+1\right)}{1+\frac{e}{2} \cos \theta_{N}} .
$$




$$
\begin{array}{r}
\text { Replacing } \frac{e}{2} \text { by } e_{N}, \text { i.e. } \\
e_{N}=\frac{e}{2},
\end{array}
$$

we obtain the standard expression of the hyerbola

$$
r_{N}\left(\theta_{N}\right)=\frac{R_{\odot}\left(e_{N}+1\right)}{1+e_{N} \cos \theta_{N}} .
$$

This equation can also directly be derived in the regular space where the eccentricity, $e_{N}$ is given by the definition equivalent to those written in Equations (2) and (20) as

$$
e_{N}=\frac{P_{N} F}{P_{N} H_{N}},
$$

in which the point $H_{N}$ is shown in Figure 3. When distances $A_{o} B_{I}$ and $B_{I} B_{N}$ are expressed by $S$ and $\tilde{x}$, i.e. $A_{o} B_{I}=s$ and $B_{I} B_{N}=\tilde{x}$, distances $P_{N} H_{I}$ and $P_{N} H_{N}$ are described as

$$
P_{N} H_{I}=s+\left[R_{\odot}-r_{N}\left(\theta_{N}\right) \cos \theta_{N}\right],
$$

and

$$
\begin{aligned}
P_{N} H_{N} & =B_{I} B_{N}+P_{N} H_{I} \\
& =\tilde{x}+s+\left[R_{\odot}-r_{N}\left(\theta_{N}\right) \cos \theta_{N}\right] .
\end{aligned}
$$

At $\theta_{N}=0$, Equation (26) yields

$$
e_{N}=\frac{R_{\odot}}{\tilde{x}+S},
$$

because of $r_{N}\left(\theta_{N}=0\right)=R_{\odot}$ and, thus,

$$
\tilde{x}=R_{\odot}\left(\frac{1}{e_{N}}-\frac{1}{e}\right),
$$

where $s=\frac{R_{\odot}}{e}$ is used. Therefore,

$$
\begin{aligned}
e_{N}= & \frac{r_{N}\left(\theta_{N}\right)}{R_{\odot}\left(\frac{1}{e_{N}}-\frac{1}{e}\right)+s+\left[R_{\odot}-r_{N}\left(\theta_{N}\right) \cos \theta_{N}\right]} \\
= & \frac{r_{N}\left(\theta_{N}\right)}{\frac{R_{\odot}}{e_{N}}+R_{\odot}-r_{N}\left(\theta_{N}\right) \cos \theta_{N}} .
\end{aligned}
$$

Solving Equation (31) with respect to $r_{N}\left(\theta_{N}\right)$, we find the result exactly identical to Equation (25).

Our result in Equation (24) explicates that the eccentricity, $e_{N}$, of the hyperbolic orbit of the corpuscle in the curved space yields just a half of the eccentricity, $e$, obtained in the regular space, when the gravitational force is directly acting on it, i.e.

$$
e_{N}=2.35 \times 10^{5} \text {. }
$$

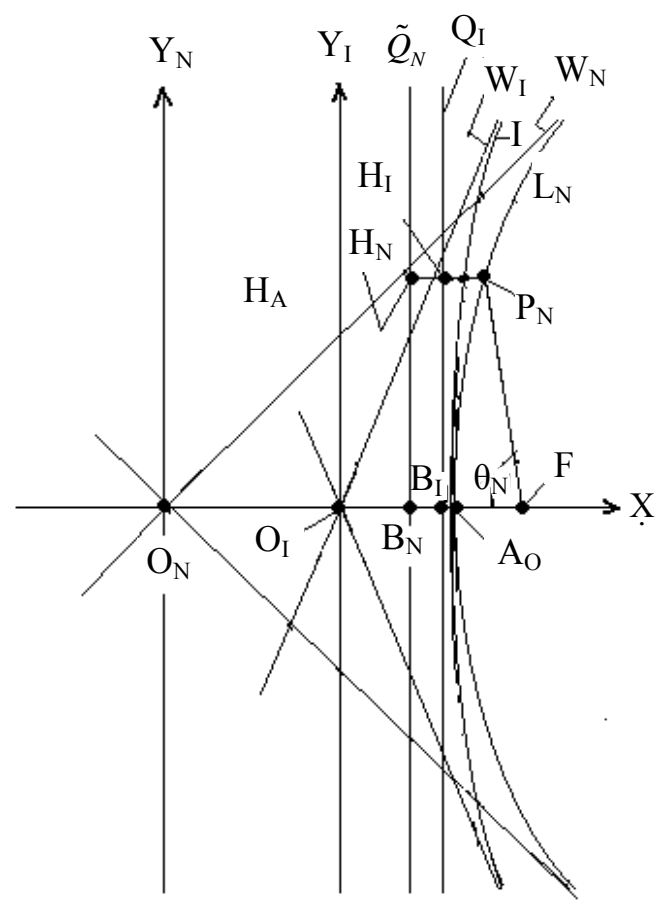

Figure 3. Paths of the corpuscle. $W_{I}$ and $W_{N}$ : asymptotes of the hyperbolae $I$ and $N . Q_{I}$ and $\tilde{Q}_{N}$ : directrices of the hyperbolae $I$ and $N$, respectively.

Thus, the Gravitational-lens effect in this case can be derived from the formula, Equation (17), provided $e$ is replaced by $e_{N}$,

$$
2 \beta_{N}=\pi-2 \arctan \sqrt{e_{N}^{2}-1}
$$

The numerical value is, now, found as

$$
2 \beta_{N}=1.755^{\prime \prime} \text {. }
$$

This value is twice of $2 \beta$ value obtained in Equation (19) and agrees with the result obtained by Einstein [4] in the GTR.

The GTR holds the concept that the photon travels along the surface of the curved space even without interacting with the source of gravitation yielding the curvature in the space, while the physical world described here is that the path of the corpuscle moving along the surface of the curved space originated from the gravitation is forced to bend by the gravitational force acting directly on it. Although the space curvature in our case is just a half of the result obtained by the GTR, the corpuscle eventually keeps moving along the same path as that predicted by the GTR.

\section{The Gravitational-Lens Effect by the Dark Matter Halo}

If the dark matter exists in surrounding of the galaxy as a halo, the gravitational-lens effect will be induced by it. In 
order to simplify the calculation, let us consider a spherical galaxy with the mass $M_{G}=5 \times 10^{11} M_{\odot}$ and the radius $R_{G}=10^{4}$ ly completely covered by the dark matter $(D M)$ with a thickness $\Delta R=R_{D M}-R_{G}=100$ ly, i.e. the radius of the sphere including the $D M$ is $R_{D M}=1.01 R_{G}$. The density of the dark matter is assumed to be as large as 10 times that of the galaxy. (i) Without any dark matter, the GLE of the corpuscle passing by the galaxy surface is found as $2 \beta=6.11^{\prime \prime}$ by using Equations (9), (24) and (33), provided $M_{\odot}$ and $R_{\odot}$ are replaced by $M_{G}$ and $R_{G}$. (ii) When the corpuscle passes beside the surface of the dark matter, the GLE is obtained as $2 \beta=7.89^{\prime \prime}$, which is calculated by replacing $M_{\odot}$ and $R_{\odot}$ by $M_{G}+M_{D M}$ and $R_{D M}$ in Equation (9). (iii) The light can penetrate into the $D M$ without scattered and sneak by the surface of the galaxy. The path of the corpuscle in the region outside the $D M$ can be calculated with Equations (9), (24) and (33) by assuming that the total mass, $M_{G}+M_{D M}$, is inside the sphere of the radius $R_{G}$. The orbit is definitely a hyperbola. On the other hand, the path of the corpuscle inside the $D M$ is able to obtain by a numerical method explained below.

Let us consider a corpuscle which starts from the galaxy surface, namely $\theta=0$ and $R_{G}$, and dashes in the dark matter until running away from the $D M$ region.

Let the corpuscle be located at $r(\theta)$ in a certain moment after it started from the galaxy surface, i.e. a distance, $r$, from and an angle, $\theta$, around the galaxy center. According to the Gaussian Theorem, the gravitational potential outside a sphere can be determined by the total mass inside the sphere. Thus, the total mass inside a sphere with the radius $r$ participates in formation of a hyperbolic orbit of the corpuscle at that moment. Remark that the location of the corpuscle, $r(\theta)$, is situated actually at the common point on the sphere surface and the hyperbolic orbit. Since the corpuscle migrates in the $D M$, it forms every moment a different sphere with a different radius and, accordingly, the total mass inside the sphere will change simultaneously. Namely, the hyperbola to which the corpuscle on the surface of the sphere formed in each moment belongs is not the same one anymore as formed in the previous moment. The trace of all these points which the corpuscle occupied every moment yields a curved line. It may not be a simple hyperbola but a slightly deviated one. Such a physical process might be explicitly described with following equations. The total mass of the system is $M=M_{G} \xi^{-1}$ with

$$
\xi^{-1}=\left[\frac{\rho_{D M}}{\rho_{G}}\left(\frac{r(\theta)}{R_{G}}\right)^{3}+\left(1-\frac{\rho_{D M}}{\rho_{G}}\right)\right],
$$

for $R_{G} \leq r(\theta) \leq R_{D M}$ where $r(\theta)$ is the distance of the corpuscle from the center of the galaxy when it is located at the angle $\theta$ around the focus. The eccentricity at this position is

$$
e_{\theta}=\frac{R_{G} c^{2}}{2 G M}-1=\left(\frac{R_{G} c^{2}}{2 G M_{G}}\right) \xi-1=e \xi-1,
$$

and, then, for $R_{G} \leq r(\theta) \leq R_{D M}$,

$$
\frac{r(\theta)}{R_{G}}=\frac{e_{\theta}+1}{1+e_{\theta} \cos \theta}=\frac{\xi e}{1+(\xi e-1) \cos \theta} .
$$

Notice that $\xi$ is also a function of $\frac{r(\theta)}{R_{G}}$. Generally, it is possible to solve Equation (37) analytically with respect to $\frac{r(\theta)}{R_{G}}$. However, it is not easy and, thus, we solve Equation (37) numerically with respect to the value of $r(\theta)$ one-by-one for each angle $\theta$ during the corpuscle passes through the $D M$ region. The orbit of the corpuscle found in this manner shows slightly deviated from a simple hyperbola, particularly in the region of the dark matter. Actually, the region of the $D M$ in which the light is moving holds only within 16 degrees around the galaxy center, i.e. $-8^{\circ} \leq \theta \leq 8^{\circ}$.

Finally, our result found for the GLE is $2 \beta=7.97$ ", which is slightly larger than that of the case (ii). This result is obviously understandable because of that the closer the orbit of the corpuscle is to the gravitational center, the larger the GLE becomes.

\section{Conclusions}

It has been shown that the GLE could be derived in the framework of Newtonian mechanics if the photon were regarded as a corpuscle with the tiny effective mass, $m_{\gamma}=\frac{h v}{c^{2}}$, which was based on the quantum theory and the Special Theory of Relativity. The factor 2 arising from the GTR has been derived by introducing the concept of the curved space due to the gravitational field and its normal reaction. Of course, all these procedures would be expediential. However, it would help senior high school and undergraduate university students to comprehend the physical structure of the GLE.

Since existence of the dark matter is not optically visible because it does not interact with the light, the GLE is known as a powerful method to observe it. The present work investigates the GLE by a spherical galaxy completely covered by the dark matter. The results are definitely in detectable range.

It is true that one can obtain informations of the dark matter by investigating the GLE.

\section{Acknowledgements}

This work has been carried out under the KAST Mentor 
Program 2010.

\section{REFERENCES}

[1] J. B. Marion, "Classical Dynamics of Particle and System," Academic Press, New York, 1970.

[2] J. G. von Soldner, Berliner Astronomisches Jahrbuch, 1801, pp. 161-172.

[3] P. Lenard, "Zur Wasserfalltheorie der Gewitter," Annalen der Physik, Vol. 370, No. 15, 1921, pp. 593-604. doi:10.1002/andp.19213701506

[4] A. Einstein, "Die Grundlage der Allgemeinen Relativittstheorie," Annalen der Physikm, Vol. 354, No. 7, 1916, pp. 769-822. doi:10.1002/andp.19163540702 